\title{
Análise de produções científicas sobre comunicação alternativa para pessoas com deficiência intelectual
}

\author{
Analysis of academic productions on alternative communication for people
} with intellectual disabilities

Análisis de producciones científicas sobre comunicación alternativa para personas con discapacidad intelectual

\author{
* Fabiana Lacerda Evaristo \\ Professora doutoranda na Universidade Federal de São Carlos (UFSCar), São Carlos, São Paulo, Brasil. \\ fabianalacerda.ufscar@gmail.com

\section{** Juliane Aparecida de Paula Perez Campos} \\ Professora doutora na Universidade Federal de São Carlos (UFSCar), São Carlos, São Paulo, Brasil. \\ jappcampos@gmail.com
}

Recebido: 4 de julho de 2017

Aprovado: 26 de julho de 2017

\section{RESUMO}

Diferentes populações podem ser beneficiadas com o sistema de comunicação alternativa, entre elas as pessoas que apresentam deficiência intelectual. Desse modo, o objetivo da pesquisa foi analisar as produções científicas encontradas sobre Comunicação Alternativa em anais de congressos científicos nacionais da área que abordam a deficiência intelectual. Para levantar as produções científicas de comunicação alternativa e estudos que envolvam pessoas com deficiência intelectual, utilizou-se como base de dados para as buscas os anais do Congresso Brasileiro de Educação Especial; do Congresso Brasileiro Multidisciplinar de Educação Especial; do Congresso Brasileiro de Comunicação Alternativa - ISAAC. Essas bases de dados foram selecionadas por abordarem estudos brasileiros numericamente significativos da área da educação especial e da comunicação alternativa. Para análise dos dados foi utilizado um roteiro de análise categorial. Os estudos analisados colaboraram para o entendimento de um cenário nacional de pesquisas dentro da área da Comunicação Alternativa. O público com deficiência intelectual encontra-se escasso nos estudos; salienta-se, com isso, a necessidade de que novas pesquisas sobre comunicação alternativa sejam desenvolvidas para pessoas com deficiência intelectual.

Palavras-chave: Educação Especial; Pesquisa bibliográfica; Comunicação alternativa. 
DOI: $10.5902 / 1984644426577$

\section{ABSTRACT}

There are several people who could be benefited with alternative communication systems, such as people with intellectual disabilities. After an academic online data base research, it was concluded that studies related to alternative communication for people with intellectual disabilities are scarce in comparison to those for other types of people. Thus, the starting point was a bibliography revision. The main purpose of this research was analyzing and describing collected academic publications about alternative communication in national congress proceedings and verifying which of them address intellectual disabilities. To achieve this, it was used data bases from the congress proceedings of the following conferences: Congresso Brasileiro de Educação Especial, Congresso Brasileiro Multidisciplinar de Educação Especial and Congresso Brasileiro de Comunicação Alternativa - ISAAC. These data bases were selected because they address numerically significant Brazilian researches about the special education area and alternative communication. Categorical analysis script was used to data analysis. The studies analyzed contributed to the understanding of a national research scenario within the area of Alternative Communication. The public with intellectual deficiency is scarce in the studies; this fact emphasizes the necessity of the development of new researches about alternative communication for people with intellectual disabilities.

Keywords: Special Education; Bibliography revision; Alternative communication.

\section{RESUMEN}

Diferentes poblaciones pueden ser beneficiadas con el sistema de comunicación alternativa, entre ellas las personas que presentan discapacidad intelectual. De este modo, el objetivo de la investigación fue analizar las producciones científicas encontradas sobre Comunicación Alternativa en anales de congresos científicos nacionales del área que abordan la discapacidad intelectual. Para levantar las producciones científicas de comunicación alternativa y estudios que involucren a personas con discapacidad intelectual, se utilizó como base de datos para las búsquedas de los anales del Congreso Brasileño de Educación Especial; del Congreso Brasileño Multidisciplinario de Educación Especial; del Congreso Brasileño de Comunicación Alternativa - ISAAC. Estas bases de datos fueron seleccionadas por abordar estudios brasileños numéricamente significativos del área de la educación especial y de la comunicación alternativa. Para el análisis de los datos se utilizó un guión de análisis categorial. Los estudios analizados colaboraron para el entendimiento de un escenario nacional de investigaciones dentro del área de la Comunicación Alternativa. El público con discapacidad intelectual se encuentra escaso en los estudios; se destaca, con ello, la necesidad de que nuevas investigaciones sobre comunicación alternativa sean desarrolladas para personas con discapacidad intelectual.

Palabras clave: Educación Especial; Investigación bibliográfica; Comunicación alternativa. 
DOI: $10.5902 / 1984644426577$

\section{Introdução}

No meio científico e clínico as características de pessoas com deficiência intelectual são encontradas no Código Internacional de Deficiências - CID-10 (1993), na American Association on Developmental Desabilities - AAMR (GARGIULO, 2008) - AAIDD (2010), e no Manual Diagnóstico e Estatístico de Transtornos Mentais DSM-V (APA, 2014).

Segundo Almeida (1988), o desenvolvimento de uma criança torna-se comprometido quando ocorrem alterações negativas durante a aquisição da linguagem. Uma em cada 200 pessoas é incapaz de comunicar-se pela fala devido a fatores neurológicos, físicos, emocionais ou cognitivos. Com isso, a linguagem oral é apenas um dos meios de comunicação (NUNES, 2003). A comunicação alternativa pode ser compreendida como uma das formas de substituir a comunicação oral dessa população.

A Comunicação Alternativa - CA apresenta várias técnicas e métodos que complementam ou substituem a linguagem oral comprometida ou ausente por meio do uso de recursos gráficos visuais e/ou gestuais (DELIBERATO; MANZINI; GUARDA, 2004). A comunicação é um fator essencial para a integração da pessoa junto da sociedade, já que a fala associada a gestos, expressões faciais e corporais caracterizam a condição humana; sendo que há formas de treinamento, estratégias e programas em CA para ensinar habilidades comunicativas.

Ziomek e Rehfeldt (2008) estudaram o uso do PECS por três alunos com deficiência intelectual. Aplicaram as fases do PECS (fase I a fase III) e dos sinais manuais. Cada participante foi ensinado em cinco mandos por itens preferidos utilizando o PECS e cinco mandos por itens preferidos usando sinais manuais. Os resultados apontaram que o PECS pode ser um sistema de comunicação alternativa viável para adultos com deficiências intelectuais que tenham pouco ou nenhum histórico de instrução sistemática e um repertório de iniciativas comunicativas limitadas.

Lima (2008) realizou um estudo com o objetivo de planejar, aplicar e avaliar um programa de intervenção sobre comunicação alternativa em um grupo inserido numa abordagem naturalística em uma escola de educação especial. Com o inicio da intervenção os participantes apresentaram desenvolvimento satisfatório quanto ao uso das figuras pictográficas, sendo que ao final todas conseguiram se comunicar por meio do intercâmbio 
de figuras. No seu estudo ficou evidente a importância da intervenção fonoaudiológica em grupo por meio da utilização de um sistema alternativo de comunicação.

Evaristo, Almeida e Boueri (2015) realizaram um estudo sobre relatos de pesquisas de comunicação alternativa, analisando especificamente o público com paralisia cerebral, no contexto brasileiro. As pesquisadoras utilizaram como base de dados o site de pesquisas Electronic Library Online, FAPESP, CNPq, BIREME / PAHO / WHO FapUnifesp - SciELO Brasil. De acordo com este levantamento, pode-se encontrar muitos estudos de comunicação alternativa, porém a maioria da população atingida é com alunos que apresentam autismo e paralisia cerebral. Foram encontrados mais trabalhos experimentais, seguidos de revisão bibliográfica e avaliação clínica.

Com a ausência de artigos nas bases de dados online, surgiu o interesse de realizarmos uma pesquisa sobre estudos de comunicação alternativa encontrados nos anais de congressos nacionais da área, dentre eles o Congresso Brasileiro de Educação Especial; Congresso Brasileiro Multidisciplinar de Educação Especial e; Congresso Brasileiro de Comunicação Alternativa - ISAAC Brasil, visto que muitos trabalhos não são publicados, restringindo-se apenas aos anais dos congressos. Com isso, apresenta relevância acadêmica e social por contribuir com a área da educação especial, ao levantar publicações importantes nos anais de eventos científicos, indicando os tipos de conhecimento que abordam essas publicações e que veiculam avanços relacionados à ciência educacional; como também será um trabalho de suporte para profissionais da área da educação especial e áreas afins, com a finalidade de colaborar com o ensino e aprendizagem das pessoas com deficiência que apresentam prejuízos na fala.

A partir dos apontamentos relatados e pelo interesse em analisar trabalhos científicos sobre comunicação alternativa para pessoas com deficiência intelectual, surgiu o seguinte questionamento: existem trabalhos científicos de comunicação alternativa em pessoas com deficiência intelectual? Se sim, qual o cenário dessas publicações nos anais dos congressos da área?

Considerando as questões elucidadas, esta pesquisa teve por objetivo analisar as produções científicas encontradas sobre Comunicação Alternativa em anais de congressos científicos nacionais da área que abordam a deficiência intelectual. 
DOI: $10.5902 / 1984644426577$

\section{Método}

A presente pesquisa caracteriza-se como um estudo de pesquisa bibliográfica, que tem como principal objetivo organizar e descrever o conhecimento já produzido em determinada área de conhecimento (LIMA; MIOTO, 2007).

A pesquisa bibliográfica permite ao investigador um aprofundamento mais amplo em aspectos teóricos, do que aqueles que poderia pesquisar diretamente. A vantagem da pesquisa bibliográfica torna-se importante quando o problema de pesquisa requer dados mais explorados (GIL, 2008).

Com o intuito de sintetizar a busca das publicações, foram utilizados os descritores dos trabalhos que apresentam nos resumos palavras relacionadas com a Comunicação Alternativa e Deficiência Intelectual, sendo: Pessoa com Deficiência Intelectual; Educação dos Deficientes Intelectuais; Comunicação; Comunicação e Educação; Educação de Pessoas com Deficiência; Related Service (Special Education); Argumentative and Alternative Communication; Intellectual Disability. Os descritores estão indexados nos dicionários de Thesaurus Brasileiro de Educação no Thesaurus do ERIC ${ }^{1}$.

Para seleção das publicações, de acordo com limite temporal, os trabalhos deveriam atender aos seguintes critérios: a) estar disponíveis e acessíveis nas edições dos anais dos congressos selecionados; b) constarem no título, resumo e palavras-chave os descritores estabelecidos; c) constarem as temáticas de Comunicação Alternativa e Deficiência Intelectual.

Como base de dados para a pesquisa foram utilizados os anais disponíveis, online e/ou livros, do Congresso Brasileiro de Educação Especial, edições: II (2005), III (2008), IV (2010), V (2012), VI (2014), VII (2016); Congresso Brasileiro Multidisciplinar de Educação Especial, edições: IV (2007), V (2009), VI (2011), VII (2013) e; Congresso Brasileiro de Comunicação Alternativa - ISAAC Brasil, edições: V (2013) e VI (2015). Essas bases de dados foram selecionadas por abordarem estudos brasileiros numericamente significativos da área da comunicação alternativa.

Primeiramente foi realizado um levantamento dos trabalhos de acordo com o título e o resumo. Após a análise dos resumos, como segundo procedimento da pesquisa, foi

1 Thesaurus Brasileiro da Educação - disponível no portal do INEP. Thesaurus do ERIC (Institute of Education Siences). 
realizada a leitura dos trabalhos completos que eram acessíveis para análise profunda sobre o interesse dos autores no tema em questão.

Após a seleção das publicações que atendiam aos critérios citados anteriormente, utilizou-se como embasamento o roteiro de Mendes, Ferreira e Nunes (2002), no qual foi adaptado para registro das informações.

Para o registro da análise dos dados, seguindo uma ordem cronológica, os trabalhos encontrados foram analisados e descritos no roteiro de análise categorial. Esse protocolo consta uma descrição mais detalhada de todas as publicações, onde constava a identificação da publicação: título, autores, evento científico, instituição e ano da publicação e; a análise descritiva da publicação: referência completa, objetivo, método utilizado na pesquisa, procedimentos metodológicos e principais resultados obtidos.

Após analisar essas categorias, qualitativamente, foi realizada uma análise que envolve a utilização de instrumentos pré-determinados que geram dados estatísticos, feita uma tabulação e agrupamento dos trabalhos de acordo com o período de publicação, a quantidade de artigos publicados ao longo de cada período e a frequência dos temas das publicações.

\section{Resultados}

Com o levantamento bibliográfico dos anais disponíveis encontramos um total de 191 trabalhos sobre Comunicação Alternativa nos congressos analisados (Congresso Brasileiro de Educação Especial, Congresso Brasileiro Multidisciplinar de Educação Especial e Congresso Brasileiro de Comunicação Alternativa). Desses artigos encontrados, 38 trabalhos abordavam temas que se relacionavam com Deficiência Intelectual.

Os artigos foram agrupados de acordo com os congressos analisados e o método de pesquisa empregado e estão organizados no quadro 1.

Quadro 1 - Artigos nacionais sobre Comunicação Alternativa para Deficiência Intelectual

\begin{tabular}{|c|c|c|c|}
\hline CONGRESSO & ARTIGOS & MÉTODO & NÚMERO \\
\hline $\begin{array}{c}\text { Congresso Brasileiro } \\
\text { de Educação }\end{array}$ & Deliberato et al. (2005) & Experimental & \\
Especial. & Deliberato e Guarda (2005) & & \\
Edições: II - 2005; III- & Moreschi e Almeida (2008) & & 7 \\
2008; IV- 2010; V - & Marba et al. (2012) & & \\
2012; VII - 2014 e; & Evaristo, Almeida e Boueri (2014) & & \\
2016- VII. & . & \\
\hline
\end{tabular}


DOI: $10.5902 / 1984644426577$

\begin{tabular}{|c|c|c|c|}
\hline & $\begin{array}{l}\text { Rodrigues, Campos e Almeida (2014) } \\
\text { Manzini et al. (2016) }\end{array}$ & & \\
\hline & $\begin{array}{l}\text { Paulo, Manzini e Deliberato (2008) } \\
\text { Lourenço, Morais e Mendes (2008) } \\
\text { Almeida et al. (2012) }\end{array}$ & Qualitativa & 3 \\
\hline & $\begin{array}{l}\text { Alves e Matsukura (2008) } \\
\text { Fiorini e Deliberato (2014) }\end{array}$ & $\begin{array}{l}\text { Qualitativa e } \\
\text { Quantitativa }\end{array}$ & 2 \\
\hline & $\begin{array}{c}\text { Mendes, Lourenço e Yokoyama (2008) } \\
\text { Vega e Grácia (2014) }\end{array}$ & Descritiva & 2 \\
\hline & $\begin{array}{c}\text { RI et al. (2012) } \\
\text { Moura et al. (2016) } \\
\text { Landin e Ramis (2014) }\end{array}$ & Estudo de caso & 3 \\
\hline $\begin{array}{l}\text { Congresso Brasileiro } \\
\text { Multidisciplinar de }\end{array}$ & $\begin{array}{c}\text { Togashi et al. (2007) } \\
\text { Quiterio (2007) } \\
\text { Araújo et al. (2013) } \\
\text { Lima, Souza e Campos (2013) }\end{array}$ & Experimental & 4 \\
\hline $\begin{array}{l}\text { Educaçao Especlal. } \\
\text { Edições: IV - 2007; V - }\end{array}$ & Borges (2007) & Qualitativa & 1 \\
\hline 2009; VI - 2011; VII - & Pozzer e Alencar (2007) & Descritiva & 1 \\
\hline 2013. & Tenor e Deliberato (2011) & \begin{tabular}{|c|} 
Qualitativa, \\
com enfoque \\
retrospectivo e \\
documental \\
\end{tabular} & 1 \\
\hline & Correia (2013) & Descritiva & 1 \\
\hline & $\begin{array}{l}\text { Alcântara et al. (2013) } \\
\text { Almeida et al. (2013) }\end{array}$ & Qualitativo & 2 \\
\hline Congresso Brasileiro & $\begin{array}{c}\text { Deliberato, Souza e Amoris (2013) } \\
\text { Quiterio e Nunes (2013) } \\
\text { Manzini et al. (2013) }\end{array}$ & Experimental & 3 \\
\hline Alternativa - ISAAC & Goldoni e Deliberato (2013) & $\begin{array}{c}\text { Revisão } \\
\text { bibliográfica }\end{array}$ & 1 \\
\hline $\begin{array}{l}\text { Edições: V - } 2013 \text { e; VI } \\
2015 .\end{array}$ & $\begin{array}{c}\text { Cardoso et al. (2015) } \\
\text { Oliveira et al. (2015) } \\
\text { Evaristo, Almeida e Boueri (2015) }\end{array}$ & $\begin{array}{l}\text { Relato de } \\
\text { experiência }\end{array}$ & 3 \\
\hline & $\begin{array}{l}\text { Lima et al. (2015) } \\
\text { Goes et al. (2015) } \\
\text { Silva et al. (2015) }\end{array}$ & $\begin{array}{l}\text { Intervenção e } \\
\text { clinico } \\
\text { qualitativa }\end{array}$ & 3 \\
\hline & Fiorini e Deliberato (2015) & $\begin{array}{c}\text { Investigação } \\
\text { social }\end{array}$ & 1 \\
\hline & & & TOTAL: 38 \\
\hline
\end{tabular}

Fonte: Elaboração própria 
Analisando os artigos de pesquisa experimental, Deliberato et al. (2005) e Deliberato e Guarda (2005), concluíram a importância da atuação em equipe para implementação de recursos de comunicação.

Moreschi e Almeida (2008) planejaram, aplicaram e avaliaram um programa de intervenção sobre Comunicação Alternativa em grupo inserido numa abordagem naturalística em uma Escola de Educação Especial. As crianças apresentaram melhora crescente quanto ao uso das figuras pictográficas, sendo que ao final do estudo todas conseguiam comunicar-se por meio do intercâmbio de figuras.

Dos estudos qualitativos, Paulo, Manzini e Deliberato (2008) desenvolveram um protocolo para avaliação de habilidades comunicativas de alunos não-falantes em ambiente escolar. Participaram deste estudo professores, fonoaudiólogas, assistentes sociais, psicólogos. Esses profissionais eram responsáveis em aplicar o protocolo com os alunos com paralisia cerebral e déficits cognitivos.

Lourenço, Morais e Mendes (2008) descreveram e analisaram as atividades desenvolvidas e o desempenho da criança com paralisia cerebral ao longo do projeto a partir da análise dos diários de campo produzidos pelos pesquisadores a campo.

Alves e Matsukura (2008) e Fiorini e Deliberato (2014), utilizaram o método qualitativo e quantitativo. Alves e Matsukura (2008) buscaram identificar a repercussão do uso da tecnologia assistiva no contexto da inclusão escolar do aluno com paralisia cerebral a partir de sua própria percepção, da percepção de seu professor e de seu familiar. Fiorini e Deliberato (2014) identificaram a interação de um aluno com síndrome de Down na rotina das atividades pedagógicas na educação infantil.

Tenor e Deliberato (2011) utilizou o método qualitativo com enfoque retrospectivo e documental. Investigaram o uso do Protocolo de Observação Comportamental (PROC) em pacientes com lesões neurológicas e comprometimentos de comunicação.

Mendes, Lourenço e Yokoyama (2008) apresentaram e descreveram o processo de composição de uma equipe composta por estudantes da área da saúde, humanas e de engenharia para a prática no projeto e apontaram possibilidades para viabilizar a formação profissional sobre a temática e a utilização de equipamento de tecnologia assistiva.

Almeida et al. (2012) descreveram a comunicação de crianças com paralisia cerebral sem condições de expressão verbal, em estimulação precoce, após a implantação do sistema de Comunicação Alternativa de alta e baixa tecnologia. 
O estudo de Marba et al. (2012) mostra-se relevante, pois a mãe não foi apenas informante das habilidades comunicativas da criança, mas teve sua participação no planejamento, implementação e confecção do recurso de comunicação suplementar e/ou alternativa.

Evaristo, Almeida e Boueri (2014) realizaram um trabalho de coensino entre a pesquisadora e a professora ao utilizar a prancha de comunicação alternativa - CA com o aluno, avaliando-o por meio das intervenções das fases do PECS-Adaptado. Rodrigues, Campos e Almeida (2014) investigaram os efeitos do PECS associado ao Vídeo Modeling acerca do desenvolvimento das habilidades de comunicação de uma aluna com síndrome de Down com necessidades complexas de comunicação. Nesses dois estudos os resultados foram o aumento das habilidades de comunicação dos participantes e a importância da implementação do sistema.

Manzini et al. (2016) capacitaram interlocutores de uma criança com paralisia cerebral para o uso de recursos de comunicação alternativa. A capacitação dos interlocutores favoreceu 0 aumento das habilidades comunicativas desta criança e promoveu modificações no comportamento da criança nos três contextos (escola, clínica e casa).

Vega e Grácia (2014) averiguaram se a assessoria em colaboração resulta em um método útil para empoderar aos profissionais em relação ao uso das comunicações simultâneas em crianças com deficiência intelectual e se tal método repercute positivamente no desenvolvimento de capacidades comunicativas infantis.

Sobre as pesquisas do tipo estudo de caso, Rl et al. (2012) demonstraram o desenvolvimento da comunicação alternativa em um paciente com a síndrome de Dandy Walker, utilizando como opção a linguagem oral, a orientação do olhar por meio de sessões de terapia fonoaudiológica em um ambiente lúdico e com atividades dirigidas.

Moura et al. (2016) relataram a prática e importância da atuação interdisciplinar entre os profissionais de terapia ocupacional e fonoaudiologia durante o processo terapêutico.

Landin e Ramis (2014) relataram uma experiência terapêutica vivida com um adolescente de 17 anos que frequentava os atendimentos realizados por uma Terapeuta Ocupacional, uma Fonoaudióloga e uma Pedagoga na Fundação Síndrome de Down, e que estava fazendo uso dos recursos de CA.

Borges (2007) identificou o vocabulário de uma criança não-falante, dentro do contexto escolar, a fim de implementar recursos de comunicação alternativa posteriormente utilizados por ela. 
DOI: $10.5902 / 1984644426577$

Pozzer e Alencar (2007) identificaram e descreveram os recursos e instrumentos de Comunicação Alternativa utilizados para subsidiar o trabalho desenvolvido junto às crianças com paralisia cerebral desprovidas de linguagem oral, bem como discorreram sobre o processo histórico, conceituaram a Paralisia Cerebral identificando os possíveis benefícios.

Togashi et al (2007) investigaram os efeitos da introdução dos recursos de comunicação alternativa na interação de alunos com paralisia cerebral e deficiência mental oralizados e não oralizados.

Quitério (2007) promoveu através do uso da Comunicação Alternativa o desenvolvimento das Habilidades Sociais em um grupo de pessoas com paralisia cerebral, a fim de que haja um maior desenvolvimento e autonomia nas interações sociais.

Araújo et al. (2013) proporcionaram uma forma de comunicação funcional a uma criança com graves limitações motoras e comunicativas.

Lima, Souza e Campos (2013) possibilitaram mais independência na comunicação de uma participante com deficiência intelectual trabalhando com a sua escrita, através de recursos da Tecnologia Assistiva.

Deliberato, Souza e Amoris (2013) analisaram a contribuição do uso das tecnologias de CA nas habilidades comunicativas de alunos com paralisia cerebral e severas dificuldades na comunicação e no entendimento, como também analisaram e o uso das tecnologias de CA para o aprendizado da leitura e escrita.

Quiterio e Nunes (2013) adaptaram o Inventário de Habilidades Sociais (DEL PRETTE; DEL PRETTE, 2005) para alunos com paralisia cerebral, não oralizados usando recursos da Comunicação Alternativa e verificaram a consistência de uma proposta de avaliação multimodal de Habilidades Sociais para pessoas com paralisia cerebral nãooralizadas.

Manzini et al. (2013) elaboraram pranchas de comunicação alternativa por meio da parceria com mães de crianças com Paralisia Cerebral não verbais. Foi possível notar nos resultados o interesse das mães em participarem da pesquisa e a dedicação no processo de elaboração das pranchas de comunicação para seus filhos.

Alcântara et al. (2013) por meio de entrevistas e observações descreveram e analisaram o processo de subjetivação e o processo de avaliação e início da implementação da Comunicação Alternativa e Ampliada (CAA) com um sujeito com paralisia cerebral. 
Almeida et al. (2013) identificaram as contribuições da wii terapia (jogo eletrônico) na reabilitação cognitiva e motora de um individuo com paralisia cerebral e apontar os benefícios da wii terapia nos atendimentos de uma criança com paralisia cerebral do tipo atetóide no NEDETA (Núcleo de Desenvolvimento de Tecnologia Assistiva e Acessibilidade).

Correia (2013) problematiza as questões de invisibilidade socioeducacional dos alunos com deficiência e descreve os processos comunicativos, mediados pela Comunicação Alternativa de alunos que apresentam severos comprometimentos motores e de fala em decorrência da Paralisia Cerebral. O aporte teórico se assenta nas contribuições de Bakhtin (2006); Vigotski (2010), Vasconcelos (1999) e Hage (2004).

Em relação aos estudos de relatos de experiência, Cardoso et al. (2015) descreveram analisaram o uso da CA como um recurso potencializador do desenvolvimento cognitivo e da comunicação de crianças com síndrome de Down.

Oliveira et al. (2015) relataram uma experiência com uma paciente que apresentava pouca interação, verbalização esporádica, dificuldades na construção de frases emitindo sempre respostas curtas. Com isso descreveram os benefícios terapêuticos da Gameterapia para a ampliação da comunicação e interação dessa paciente com paralisia cerebral.

Evaristo, Almeida e Boueri (2015) avaliaram as habilidades comunicativas de um aluno com paralisia cerebral e déficits na oralidade por meio do Sistema de Comunicação por Troca de Figuras - PECS-Adaptado. A introdução do sistema PECS-Adaptado trouxe sucesso e melhora para a comunicação do aluno, como também melhorou o entendimento com seus interlocutores. Observou-se também a importância em desenvolver programas de comunicação alternativa com alunos que não apresentam a fala oral.

Foi possível localizar também estudos de revisão bibliográfica, de intervenção e clínicos qualitativos.

Goldoni e Deliberato (2013) realizaram uma busca a fim de identificarem em manuais, guias e folhetos de orientação à família do aluno com paralisia cerebral usuário de sistemas alternativos de comunicação por meio da literatura. Foi realizada busca em bases de dados nacionais e internacionais, revistas específicas da área e no banco de teses e dissertações da CAPES e da SCIRUS publicados no período de 2001 a 2013. As informações foram coletadas utilizando um protocolo para avaliar a forma e conteúdo dos materiais 
selecionados, proposto por Ferreira (2006). Com o levantamento, não foram identificados artigos na temática estudada entre as diferentes bases de dados pesquisadas.

Lima et al. (2015) testaram hardware e software de baixo custo para uso da Comunicação Alternativa e Ampliada em pessoas com paralisia cerebral.

Goes et al. (2015) investigaram como a avaliação neurofuncional pode contribuir na seleção de mecanismos e ferramentas da Comunicação Alternativa com sujeitos entre cinco e vinte anos de idade sem oralidade ou com dificuldades significativas.

Silva et al. (2015) apresentaram a experiência na descrição das habilidades comunicativas de usuários com Paralisia Cerebral assistidos no Núcleo de Desenvolvimento de Tecnologia Assistiva e Acessibilidade (NEDETA).

Fiorini e Deliberato (2015) replicaram um estudo apresentado no Congresso Brasileiro de Educação Especial. Identificaram a interação de um aluno com síndrome de Down na rotina das atividades pedagógicas na educação infantil. Os resultados indicaram que o participante do estudo manteve a atenção nas atividades propostas, principalmente no interlocutor responsável pelo direcionamento da tarefa, confirmando a necessidade dessa função para a manutenção da interação.

Após a análise qualitativa dos estudos, realizou-se uma análise quantitativa por meio de gráficos.

\section{Congresso Brasileiro de Educação Especial - CBEE}

O gráfico 1 representa o total de trabalhos sobre Comunicação Alternativa e o total desses trabalhos que abordam o tema de deficiência intelectual, no Congresso Brasileiro de Educação Especial, realizado na Universidade Federal de São Carlos nos anais dos anos de 2005, 2010, 2012, 2014 e 2016. 
DOI: $10.5902 / 1984644426577$

Gráfico 1 - Total de trabalhos de CA e CA para DI no CBEE

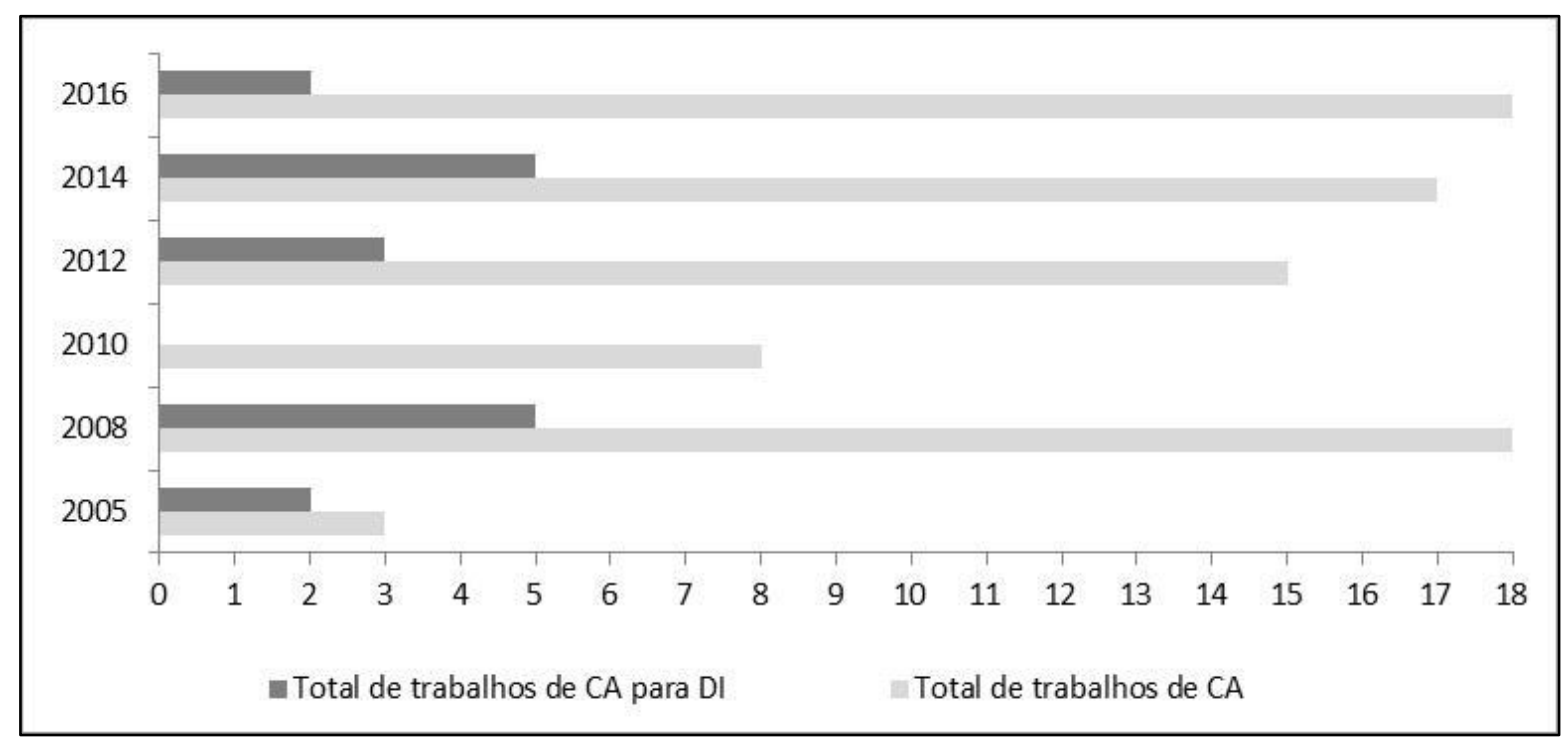

Legenda: $\mathrm{CA}$ = Comunicação Alternativa; $\mathrm{DI}=$ Deficiência Intelectual

De acordo com o gráfico 1 o total de trabalhos de CA nos respectivos anos analisados foram 71 trabalhos. Com a análise desses trabalhos os que envolviam pessoas com DI foram 17 trabalhos.

Nota-se que de 2005 para 2008 houve um aumento de 3 trabalhos que envolviam pessoas com DI, como também houve um aumento potencial de trabalhos sobre CA. Porém em 2010 não foram publicados nenhum trabalho para o público com DI.

De 2012 até 2016 houve uma oscilação nos trabalhos para o público em questão, porém em 2016 de 18 trabalhos publicados sobre CA, apenas 2 trabalhos estudados abordavam a DI.

\section{Congresso Brasileiro Multidisciplinar de Educação Especial - CBMEE}

O gráfico 2 representa o total de trabalhos sobre CA e o total desses trabalhos que abordam o tema de DI no CBMEE realizado na Universidade de Londrina nos anais dos anos de 2007, 2009, 2011 e 2013. 
DOI: $10.5902 / 1984644426577$

Gráfico 2 - Total de trabalhos de CA e CA para DI no CBMEE

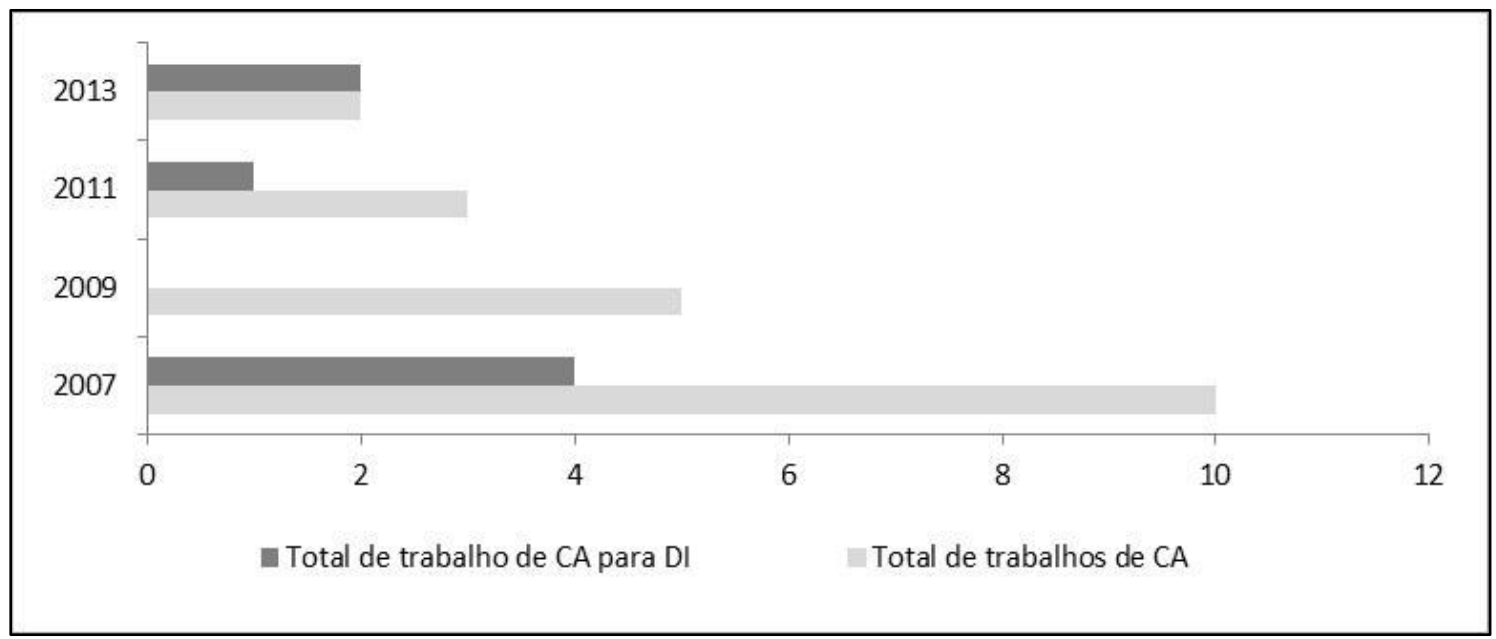

Legenda: $\mathrm{CA}$ = Comunicação Alternativa; $\mathrm{DI}$ = Deficiência Intelectual

De acordo com o gráfico 2, dos 20 trabalhos sobre CA, 7 trabalhos abordavam a DI. Em comparação ao número de trabalhos sobre Comunicação Alternativa, o público com Deficiência Intelectual é relativamente baixo.

Observa-se que em 2009 houve uma queda de publicações e não foi publicado nenhum trabalho que envolvesse o público com deficiência intelectual. Em 2011 o número de trabalhos sobre CA também diminuiu, porém houve 1 trabalho sobre DI. Especificamente em 2013 todos os trabalhos de CA abordavam a DI.

\section{Congresso Brasileiro de Comunicação Alternativa - ISAAC Brasil}

O gráfico 3 representa o total de trabalhos sobre CA e o total desses trabalhos que abordam o tema de DI no ISAAC-Brasil nos anais encontrados dos anos de 2013 e 2015. 
DOI: $10.5902 / 1984644426577$

Gráfico 3 - Total de trabalhos de CA e CA para DI no CBEE

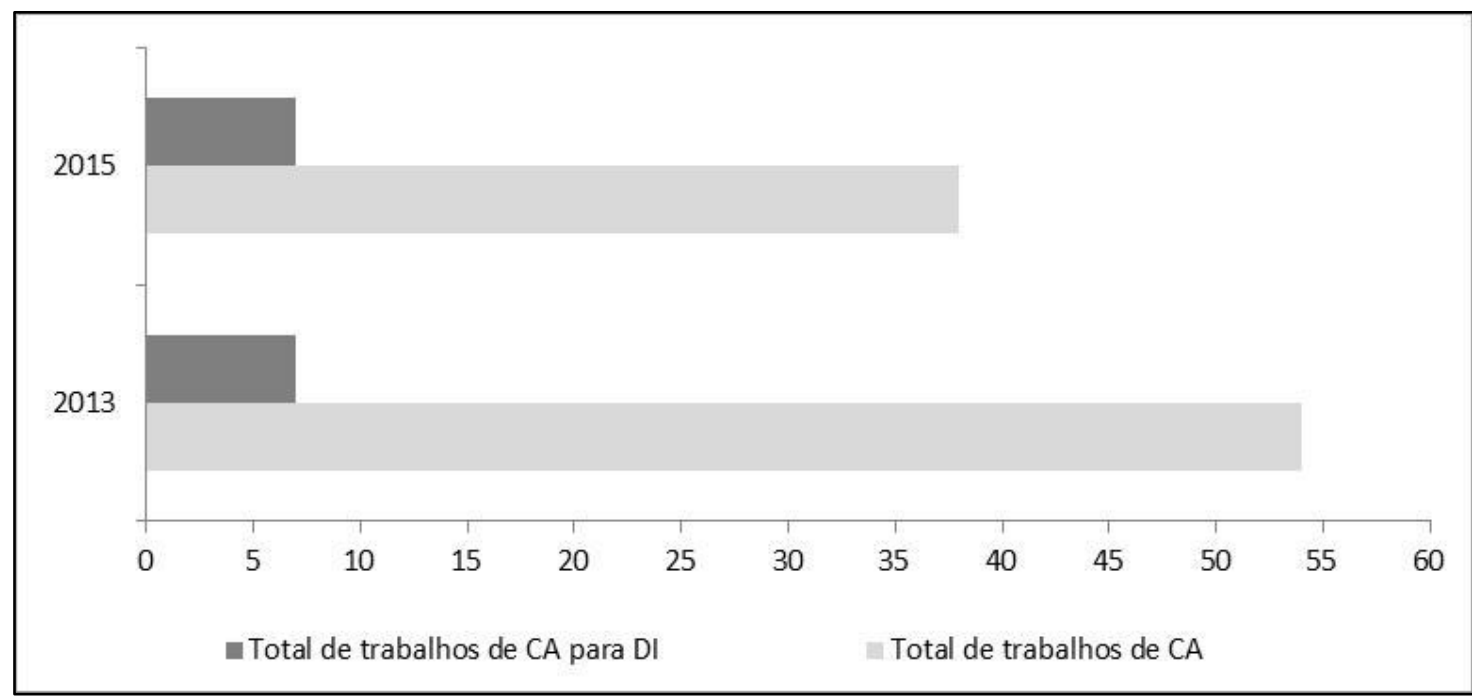

Legenda: $\mathrm{CA}$ = Comunicação Alternativa; DI = Deficiência Intelectual

De acordo com o gráfico 3, dos 91 trabalhos sobre CA, 14 trabalhos abordavam a DI.

Por se tratar de um congresso específico da área da Comunicação Alternativa, o número de trabalhos que abordam a temática de DI é baixo.

\section{Discussão}

De maneira geral, os resultados das pesquisas demonstram que a Comunicação Alternativa foi eficiente para os objetivos expostos, desde a importância do trabalho em equipe, a capacitação de interlocutores e principalmente, para a aquisição de habilidades comunicativas de pessoas com deficiência intelectual. Porém, com os resultados, o tema "Comunicação Alternativa" apresenta uma quantidade razoável de artigos pesquisados no Brasil, mas pode-se observar que a maioria dos autores abordados são os mesmos, e o público de Deficiência Intelectual ainda não é o foco da maioria dos trabalhos.

Os artigos abordados no quadro 1 trabalharam com o público de deficiência intelectual, porém com metodologias diferentes, sendo estudos experimentais, qualitativos, quantitativos, descritivos, estudos de caso, relatos de experiência, intervenção, clínico qualitativo, revisão bibliográfica e investigação social. O método mais empregado nos trabalhos foi a pesquisa experimental.

Foi possível analisar nos trabalhos a importância da atuação interdisciplinar em equipe para implementação de recursos de comunicação, da aceitação do contato fisíco dos 
DOI: $10.5902 / 1984644426577$

alunos com os profissionais (DELIBERATO et al., 2005; DELIBERATO e GUARDA 2005; MOURA et al., 2016).

Damiani (2008) descreve a importância do trabalho em conjunto, pois, assim, as pessoas podem partilhar ideias, histórias, experiências e conhecimento, podendo atingir um resultado mais rico e complexo do que o derivado do trabalho individual. Além disso, a parceria oferece aos profissionais maiores oportunidades de enriquecerem sua maneira de pensar, agir e solucionar problemas, tendo mais chances de sucesso nas tarefas pedagógicas.

A implementação do sistema de Comunicação Alternativa beneficia o aumento das habilidades comunicativas dos participantes (MORESCHI e ALMEIDA, 2008; TOGASHI et al., 2007; ARAÚJO et al., 2013) para a aprendizagem da leitura e da escrita (DELIBERATO, SOUZA e AMORIS, 2013; LIMA, SOUZA e CAMPOS, 2013; LANDIN e RAMOS, 2014) como para que haja um maior desenvolvimento e autonomia nas interações sociais (QUITÉRIO, 2007; RI et al., 2012; QUITERIO e NUNES, 2013).

Deliberato (2009) e Sameshima (2011) demonstraram que os recursos e procedimentos da área de comunicação alternativa vêm favorecendo não só a ampliação das habilidades comunicativas, mas também contribuindo para a aquisição e desenvolvimento da linguagem e aprendizagem escolar do aluno com deficiência.

A capacitação de interlocutores também foi um ponto abordado nas pesquisas. Mostra-se relevante ao capacitar as mães como interlocutores imediatos, a participarem no planejamento, implementação e confecção dos recursos de Comunicação Alternativa (MARBA et al., 2012) ao favorecer o aumento das habilidades comunicativas dos participantes e promover mudanças no comportamento (EVARISTO, ALMEIDA e BOUERI, 2014; RODRIGUES, CAMPOS e ALMEIDA, 2014; MANZINI et al. 2016).

A qualidade da capacitação dos interlocutores é uma das variáveis mais importantes para o bom andamento do processo ensino-aprendizagem (FREITAS, 1995). É preciso, então, que o corpo docente reveja suas concepções e reconstrua seus objetivos para que esses incluam a aceitação das diferenças. Dessa maneira, poder-se-á "buscar, em cada aluno, as suas qualidades desejáveis, em vez de acentuar ainda mais suas inadequações para determinadas tarefas" (LEITE, 1981, p. 245).

Nos estudos qualitativos estudados foram desenvolvidos protocolos de avaliação de habilidades comunicativas (PAULO, MANZINI e DELIBERATO, 2008) análise das atividades desenvolvidas (LOURENÇO, MORAIS e MENDES, 2008) por meio de 
entrevistas, observações e análise de diários de campo (BORGES, 2007; ALCÂNTARA et al., 2013) análise da implementação de jogos eletrônicos (ALMEIDA et al., 2013) análise da repercussão do uso da Tecnologia Assistiva (ALVES e MATSUKURA, 2008; FIORINI e DELIBERATO, 2014) método qualitativo com enfoque retrospectivo e documental, com uso do Protocolo de Observação Comportamental (TENOR e DELIBERATO, 2011).

Os estudos descritivos abordados descreveram os recursos e instrumentos de Comunicação Alternativa para crianças desprovidas de linguagem oral (POZZER e ALENCAR, 2007), apresentaram possibilidades de viabilizar a formação profissional sobre a temática da tecnologia assistiva (MENDES, LOURENÇO e YOKOYAMA 2008), expuseram processos comunicativos, mediados pela Comunicação Alternativa de alunos que apresentam severos comprometimentos motores e de fala em decorrência da Paralisia Cerebral (CORREIA, 2013), e comunicações simultâneas (VEGA e GRÁCIA, 2014).

Apesar das diferenças dos procedimentos e resultados dos estudos, demonstraram a mudança comportamental dos participantes diante da aquisição da comunicação alternativa, com exceção do estudo de Goldoni e Deliberato (2013) que realizaram uma revisão bibliográfica, a fim de identificarem guias, folhas ou manuais de orientações para famílias de usuários de Comunicação Alternativa. Diante das buscas, não foram localizados nenhum material ligado à temática estudada.

Há algumas limitações que devem ser consideradas nesta revisão. Houve dificuldade em encontrar os anais dos congressos selecionados que estavam disponíveis. $O$ Congresso de Comunicação Alternativa - ISAAC Brasil é um congresso considerável para a área em questão, está em sua VII edição, e de todos os anais só foi possível localizar os anais dos anos de 2013 (V edição) e de 2015 (VI edição). Há princípio pensamos em analisar os estudos internacionais da área, porém houve dificuldade em localiza-los. Por isso, embora os esforços de pesquisa detalhados tenham sido feitos seguindo critérios específicos, é possível que estudos relevantes não tenham sido incluídos na presente revisão.

A Comunicação Alternativa é uma forma alternativa de comunicação para pessoas que possuem comprometimentos na fala oral. Em geral, os estudos abordados nesta revisão sobre programas de intervenção voltados para o ensino de habilidades comunicativas, capacitação de diferentes interlocutores, interações sociais, entre outros objetivos, são eficazes e trazem relevância aos efeitos positivos que estes programas beneficiam. 
DOI: $10.5902 / 1984644426577$

\section{Conclusões}

Pode-se analisar nos estudos que a maioria dos trabalhos utilizavam o método experimental. Enfatiza-se com isso a importância dos trabalhos de Comunicação Alternativa serem implementados pelo método experimental.

As revisões dos estudos demonstraram que o público com deficiência intelectual na área da comunicação alternativa encontra-se escasso de acordo com os gráficos apresentados, e os estudos publicados nesses anais não foram encontrados na base de dados online. Salienta-se, com isso, a necessidade de que novas pesquisas sobre comunicação alternativa sejam desenvolvidas para pessoas com deficiência intelectual.

Os estudos descritos colaboraram para o entendimento de um cenário nacional de pesquisas dentro da área da Comunicação Alternativa. Com isso, considera-se que o estudo respondeu aos objetivos propostos podendo acrescentar conhecimento ao que vem sendo produzido na área.

\section{Referências}

AAIDD. Intellectual disability: definition, classification and systems of supports. 11th ed. Washington (DC): American Association on Intellectual and Developmental Disabilities, 2010.

ALCÂNTARA, J. N. et al. A clínica da subjetividade e a implementação da comunicação alternativa e ampliada: a construção do sujeito da linguagem. In: V Congresso Brasileiro de Comunicação Alternativa, 5, 2013, Gramado. Gramado. Anais...ISAAC Brasil, 2013, p. 1 - 16.

ALENCAR, G. A. R. Paralisia Cerebral e Comunicação Alternativa: Definindo conceitos. In: IV CONGRESSO BRASILEIRO MULTIDISCIPLINAR DE EDUCAÇÃO ESPECIAL. 4., 2007, Londrina. Anais...Londrina: UEL, 2007. p. 1-8.

ALMEIDA, M. A. O ensino funcional da linguagem para indivíduos especiais. In: REUNIÃO ANUAL DE PSICOLOGIA, 1988. Ribeirão Preto. Anais da Sociedade de Psicologia de Ribeirão Preto, 1988. p. 677-684.

ALMEIDA, P. et al. A Comunicação Alternativa e Paralisia Cerebral: estimulação precoce - crianças de 2 a 5 anos. In: V CONGRESSO BRASILEIRO DE EDUCAÇÃO ESPECIAL, VIII ENCONTRO DA ASSOCIAÇÃO BRASILEIRO DE PESQUISADORES EM EDUCAÇÃO ESPECIAL, v.5, 2008, São Carlos. Anais...São Carlos: ABPEE, 2012. 
ALMEIDA, H. C. et al. A Análise das contribuições do WII terapia no desenvolvimento motor e cognitivos de um adolescente com paralisia cerebral.. In: V Congresso Brasileiro de Comunicação Alternativa, 5, 2013, Gramado. Gramado. Anais...ISAAC Brasil, 2013, p. $1-8$.

ALVES, A. C. J.; MATSUKURA, T. S. Os recursos de Tecnologia Assistiva na inclusão escolar de alunos com Paralisia Cerebral. In: III CONGRESSO BRASILEIRO DE EDUCAÇÃO ESPECIAL, III ENCONTRO DA ASSOCIAÇÃO BRASILEIRO DE PESQUISADORES EM EDUCAÇÃO ESPECIAL, 3., 2008, São Carlos. Anais... São Carlos, 2008. p. 151-151.

APA (American Psychiatric Association ). DSM-5: Manual Diagnóstico E Estatístico De Transtornos Mentais. 5. ed. Porto Alegre: Artmed, 2014.

ARAÚJO, R. T. F. et al. Comunicação Alternativa e/ou Suplementar na intervenção terapêutica ocupacional de paciente com síndrome de Werdnig Hoffmann. In: VII CONGRESSO BRASILEIRO MULTIDISCIPLINAR DE EDUCAÇÃO ESPECIAL. 7., 2013, Londrina, Paraná. Anais... Londrina: UEL, 2013. p. 1-9.

BAKHTIN, Mikhail (Volochínov). Marxismo e filosofia da linguagem. São Paulo: Hucitec, 2006.

BONDY, A.; FROST, L. The Picture Communication Symbols. Stillwater , Minnesota: Mayer-Johnson Co. vol. IV, 1981.

The Picture Communication Symbols. Stillwater, Minnesota: Mayer-Johnson Co. vol. V, 1985.

The Picture Communication Symbols. Stillwater, Minnesota: Mayer-Johnson Co. vol. VI, , 1992.

PECS: The Pictura Exchange Communication System. Cherry Hill, NJ: Pyramid Educational Consultants, Inc. 1994.

Manual do usuário Boardmaker. Porto Alegre: Click - Recursos Tecnológicos para Educação, Comunicação e Facilitação, 2005.

The picture exchange communication system. Topics in Language Disorders, v.19, p. 373-390, 1998.

BORGES, A. C. A Identificação do vocabulário no contexto escolar para implementação da Comunicação Alternativa em uma criança não-falante. In: IV CONGRESSO BRASILEIRO MULTIDISCIPLINAR DE EDUCAÇÃO ESPECIAL. 4., 2007, Londrina. Anais... Londrina: UEL, 2007, p. 1-8.

BLISS, C. Semantography (Blissymbolics). Sydney: Semantography Publications, 1965. 
CARDOSO, M. F. et al. COMUNICAÇÃO SUPLEMENTAR E/OU ALTERNATIVA NO ATENDIMENTO ÀSPESSOAS COM SÍNDROME DE DOWN: RELATO DE EXPERIÊNCIA. In: VI Congresso Brasileiro de Comunicação Alternativa, 6, 2015, Campinas-SP. Campinas. Anais...ISAAC Brasil, 2015, p. 1 - 11.

CLASSIFICAÇÃO DOS TRANSTORNOS MENTAIS E DO COMPORTAMENTO DA CID-10. Descrições clínicas e diretrizes diagnósticas. BRASÍLIA: MEC/CORDE, 1993.

CERVO, A. L.; BERVIAN, P. A. Metodologia científica. 5. ed. São Paulo: Prentice Hall, 2002.

CORREIA, V. G. de P.. A comunicação alternativa como via de acesso á inclusão: visibilizando os potenciais comunicativos de uma aluna com paralisia cerebral. In: V Congresso Brasileiro de Comunicação Alternativa, 5, 2013, Gramado. Gramado. Anais...ISAAC Brasil, 2013, p. 1 - 15.

CHUN, R. Y. S.; MOREIRA, E. C.; DALLAQUA, G. B. Análise da produção dos Congressos Brasileiros de Comunicação Alternativa: o estado da arte da CSA no Brasil. In: IV CONGRESSO BRASILEIRO DE COMUNICAÇÃO ALTERNATIVA: compartilhando experiências. Rio de Janeiro. Anais...Rio de Janeiro: UERJ, 2011, p. 1-5. 1 CD-ROM.

DAMIANI, M. F. Entendendo o trabalho colaborativo em educação e revelando seus benefícios. Educar, Editora UFPR, Curitiba, n. 31, 2008, p. 213-230.

DEL PRETTE, Z.A.P.; DEL PRETTE, A. Sistema Multimídia de Habilidades Sociais de Crianças: (SMHSC-Del-Prette) manual. São Paulo: Casa do Psicólogo, 2005. 99p.

DELIBERATO, D.; MANZINI, E.; GUARDA, N. S. A implementação de recursos suplementares de comunicação: participação da família na descrição de comportamentos comunicativos dos filhos. Revista Brasileira de Educação Especial, Marília, v.10, n.2, p. 217-240, 2004.

DELIBERATO, L. M. P. et al. Procedimentos de implementação de recursos de Comunicação Alternativa para uma aluna com Paralisia Cerebral: Cuidados com vocabulário e acesso Motor. In: II CONGRESSO BRASILEIRO DE EDUCAÇÃO ESPECIAL, II ENCONTRO DA ASSOCIAÇÃO BRASILEIRO DE PESQUISADORES EM EDUCAÇÃO ESPECIAL, 2., 2005, São Carlos. Anais... São Carlos: ABPEE, 2005, p. 213-214.

DELIBERATO, D. ALVES, V. A.; GUARDA, N. S. Comunicação Alternativa e Suplementar no atendimento do aluno com multideficiência. In: II CONGRESSO BRASILEIRO DE EDUCAÇÃO ESPECIAL, II ENCONTRO DA ASSOCIAÇÃO BRASILEIRO DE PESQUISADORES EM EDUCAÇÃO ESPECIAL, 2., 2005, São Carlos. Anais... São Carlos: ABPEE, 2005, p. 215-215.

DELIBERATO, D.. Comunicação alternativa na escola: habilidades comunicativas e 0 ensino da leitura e escrita. In: DELIBERATO, D.; GONÇALVES, M. J.; MACEDO, E.C. 
(Org). Comunicação alternativa: teoria, prática, tecnologias e pesquisa. São Paulo: Memnon Edições Científicas, 2009. p. 235-243.

DELIBERATO, D.; SOUZA, F. P.; AMORIS, E. V. N.. Tecnologias da comunicação alternativa: recursos e procedimentos para alunos com paralisia cerebral. In: V Congresso Brasileiro de Comunicação Alternativa, 5, 2013, Gramado. Gramado. Anais...ISAAC Brasil, 2013, p. 1 - 19.

EVARISTO, F. L.; BOUERI, I. Z.; ALMEIDA, M. A. Implicações do programa PECSAdaptado no repertório comunicativo de um aluno com paralisia cerebral. In: VI CONGRESSO BRASILEIRO DE EDUCAÇÃO ESPECIAL, IX ENCONTRO DA ASSOCIAÇÃO BRASILEIRO DE PESQUISADORES EM EDUCAÇÃO ESPECIAL, v.6, 2014, São Carlos. Anais... São Carlos: ABPEE, 2014. p. 1-15.

EVARISTO, F. L; ALMEIDA, M. A.; BOUERI, I. Z. Avaliação das habilidades comunicativas de um aluno com paralisia cerebral. In: CONGRESSO BRASILEIRO DE COMUNICAÇÃO ALTERNATIVA - ISAAC Brasil. Campinas-Sp. Anais... Universidade Estadual de Campinas, n.5, out-2015.

FIORINI, B. S.; DELIBERATO, D. HABILIDADES DE INTERAÇÃO DE UM ALUNO COM SÍNDROME DE DOWN NA EDUCAÇÃO INFANTIL. In: VI CONGRESSO BRASILEIRO DE EDUCAÇÃO ESPECIAL, IX ENCONTRO DA ASSOCIAÇÃO BRASILEIRO DE PESQUISADORES EM EDUCAÇÃO ESPECIAL, v.5, 2014, São Carlos. Anais... São Carlos: ABPEE, 2014.

FIORINI, B. S.; DELIBERATO, D. HABILIDADES DE INTERAÇÃO DE UM ALUNO COM SÍNDROME DE DOWN NA EDUCAÇÃO INFANTIL. In: VI Congresso Brasileiro de Comunicação Alternativa, 6, 2015, Campinas-SP. Campinas. Anais...ISAAC Brasil, 2015, p. $1-15$.

FERREIRA, G. C. Programa de educação familiar continuada em linguagem: orientação a pais de crianças com atrasos globais do desenvolvimento. Dissertação (mestrado em educação) Curso de Pós-graduação em Educação, Universidade Estadual Paulista, UNESP, Marília, 2006.

FREITAS, K. S. Importância da Teleducação na Capacitação De Professores. Sitientibus, Feira de Santana, n.11, 1995, p.113-119.

GARGIULO, R. M. Individuals with mental retardation or intellectual disabilities. In: Special Education in Contemporary Society: an Introduction to Exceptionality. Beverly Hills: Sage Publications Inc., 2008. p. 3-62.

GIL, A. C. Métodos e técnicas de pesquisa social. 6ed. São Paulo: Atlas, 2008.

GOES, U. M. et al. AVALIAÇÃO NEUROFUNCIONAL COMO NORTEADORA DA SELEÇÃO DE FERRAMENTAS DE CAA EM SUJEITOS COM PARALISA CEREBRAL. In: VI Congresso Brasileiro de Comunicação Alternativa, 6, 2015, Campinas-SP. Campinas. Anais...ISAAC Brasil, 2015, p. 1 - 11. 
DOI: $10.5902 / 1984644426577$

GOLDONI, N. I.; DELIBERATO, D. Orientação familiar do aluno com paralisia cerebral usuário de comunicação suplementar alternativa : revisão bibliográfica. In: V Congresso Brasileiro de Comunicação Alternativa, 5, 2013, Gramado. Gramado. Anais...ISAAC Brasil, 2013, p. 1 - 16.

HAGE, S. R. de V.. Avaliando a linguagem na ausência da oralidade. Bauru, SP: EDUSC, 2004.

JANNUZZI, G.M. Cresce o engajamento da sociedade civil e política nesta educação. In: A educação do deficiente no Brasil: dos primórdios ao início do século XXI. Campinas: Autores Associados, 2004. p. 67-136.

LANDIN, S.; RAMOS, E. O uso de recursos da Comunicação Suplementar Alternativa por um adolescente com Síndrome de Down. In: VI CONGRESSO BRASILEIRO DE EDUCAÇÃO ESPECIAL, IX ENCONTRO DA ASSOCIAÇÃO BRASILEIRO DE PESQUISADORES EM EDUCAÇÃO ESPECIAL, v.5, 2014, São Carlos. Anais... São Carlos: ABPEE, 2014.

LAKATOS, E. M; MARCONI, M. A. Fundamentos de Metodologia Científica. 7. ed. São Paulo: Atlas, 2010.

LEBLANC, J. M. El curriculum funcional en la educación de la persona com retardo mental. In: SIMPÓSIO INTERNATIONAL COANIL, 1991, Santiago, Chile. Proceedings... Santiago, 1991. p.10.

LEITE, D. M. Educação e relações interpessoais. In: PATTO, M.H.S. Introdução à Psicologia Escolar. São Paulo: TAQ. 1981, p. 234-257.

LIMA, T. C. S. de.; MIOTO, R. C. T. Procedimentos metodológicos na construção do conhecimento científico: a pesquisa bibliográfica. Rev. Katál. Florianópolis v. 10 n. esp. p. 37-45, 2007.

LIMA, C. S. Eficácia de um programa de comunicação alternativa aplicada a grupos de escolares com deficiência intelectual. 2008. 226f. Dissertação (Mestrado em Educação Especial) - Programa de Pós Graduação em Educação Especial. Universidade Federal de São Carlos, São Carlos, 2008.

LIMA, T. A. V. H.; SOUZA, N. E.; CAMPOS, R. L. S. A Comunicação Alternativa e Ampliada no auxilio da independência de uma aluna. In: VII CONGRESSO BRASILEIRO MULTIDISCIPLINAR DE EDUCAÇÃO ESPECIAL. 7., 2013, Londrina, Paraná. Anais... Londrina: UEL, 2013.

LIMA, M. V. A. et al. MÉTODO DE ACOMPANHAMENTO PARA SUJEITOS COM PARALISIA CEREBRAL USUÁRIOS DE COMUNICAÇÃO ALTERNATIVA E AMPLIADA. In: VI Congresso Brasileiro de Comunicação Alternativa, 6, 2015, Campinas-SP. Campinas. Anais...ISAAC Brasil, 2015, p. 1 - 9.

LOURENÇO, G. F.; MORAES, N. F. R.; MENDES, E. G.O uso da Tecnologia Assistiva como ferramenta complementar para alfabetização de alunos com Paralisia Cerebral: 
DOI: $10.5902 / 1984644426577$

a distância entre sala de recursos e sala comum. In: III CONGRESSO BRASILEIRO DE EDUCAÇÃO ESPECIAL, III ENCONTRO DA ASSOCIAÇÃO BRASILEIRO DE PESQUISADORES EM EDUCAÇÃO ESPECIAL, 3., 2008, São Carlos. Anais... São Carlos: ABPEE, 2008, p. 151-151.

LUCKASSON, R. et al. Mental Retardation - Definition Classification, and Systems of Supports. 10ed. Washington (DC): American Association on Mental Retardation, 2002.

MANZINI, M. G. et al. Processo de elaboração de pranchas de comunicação alternativa : parceria com mães de crianças não verbais. In: V Congresso Brasileiro de Comunicação Alternativa, 5, 2013, Gramado. Gramado. Anais...ISAAC Brasil, 2013, p. $1-16$.

MANZINI, M. G. et al. Capacitação de diferentes interlocutores para usos de recursos de comunicação alternativa: uma proposta inclusiva. In: VII CONGRESSO BRASILEIRO DE EDUCAÇÃO ESPECIAL, IX ENCONTRO DA ASSOCIAÇÃO BRASILEIRO DE PESQUISADORES EM EDUCAÇÃO ESPECIAL, 7, 2016 São Carlos. Anais da UFSCAR/PPGEEs; ABPEE, 2016, p. 1-15.

MAHARAJ, S. Pictogram ideogram communication. Regina-Canada: The George Reed Foundation for the Handicapped, 1980.

MANZO, A. J. Manual para la preparación de monografias: una guía para presentar informes y tesis. Humanitas: Buenos Aires, 1971.

MENDES, E. G.; FERREIRA, J. R.; NUNES, L. R. P. Análise crítica das teses e dissertações sobre Educação Especial nas áreas de Educação e Psicologia PRODICS IV. Relatório Final de Pesquisa. FAPESP. 2002.

MENDES, E. G.; LOURENÇO, G. F.; YOKOYAMA, M. H. Projeto Alta TA e inclusão: desenvolvendo o voluntariado e a multidisciplinariedade na implementação de recursos de alta tecnologia assistiva com alunos com Paralisia Cerebral. In: III CONGRESSO BRASILEIRO DE EDUCAÇÃO ESPECIAL, III ENCONTRO DA ASSOCIAÇÃO BRASILEIRO DE PESQUISADORES EM EDUCAÇÃO ESPECIAL, 3., 2008, São Carlos. Anais da UFSCAR/PPGEEs: ABPEE, 2008, p. 152-152.

MOURA, F. G. et al. A intervenção interdisciplinar de pacientes com Síndrome de Down. In: VII CONGRESSO BRASILEIRO DE EDUCAÇÃO ESPECIAL, IX ENCONTRO DA ASSOCIAÇÃO BRASILEIRO DE PESQUISADORES EM EDUCAÇÃO ESPECIAL, 7, 2016 São Carlos. Anais da UFSCAR/PPGEEs; ABPEE, 2016, p. 1-13.

MORESCHI, C. L.; ALMEIDA, M. A. Eficácia de um programa de Comunicação Alternativa aplicado a grupo de escolares com Deficiência Intelectual. In: III CONGRESSO BRASILEIRO DE EDUCAÇÃO ESPECIAL, III ENCONTRO DA ASSOCIAÇÃO BRASILEIRO DE PESQUISADORES EM EDUCAÇÃO ESPECIAL, 3., 2008, São Carlos. Anais da UFSCAR/PPGEEs; ABPEE, 2008, p. 144-144. 
DOI: $10.5902 / 1984644426577$

MORESCHI, L.C. Perfil comunicativo de usuário de Sistemas de Comunicação Alternativa na Interação com Diferentes Parceiros. 2012. 182 f. Tese (Doutorado em Educação Especial) - Programa de Pós Graduação em Educação Especial. Universidade Federal de São Carlos, São Carlos. 2012.

NUNES, D.R.; NUNES, L.R. Efeitos dos procedimentos naturalísticos no processo de aquisição de linguagem através de sistema pictográfico em criança autista. Em L.R. Nunes (Org.), Favorecendo o desenvolvimento da comunicação em crianças e jovens com necessidades educacionais especiais. Rio de Janeiro: Dunya, p. 125$141,2003$.

NUNES, L. R. O. de P.; et al. Comunicação Alternativa e Autismo: isto dá samba? In: NUNES, L. R. O. de P; PELOSI, M. B.; GOMES, M. R. Um Retrato de Comunicação Alternativa no Brasil. Rio de Janeiro: Estúdio Gráfico e Papéis, 2007, 320p.

NUNES, D.R.P. Introdução. In: MANZINI, E.J.; MARQUEZINE, M.C.; TANAKA, E.D.O.; FUJISAWAM, D.S.; BUSTO, R.M. Linguagem e comunicação alternativa. Londrina: ABPEE, p. 1-8, 2009.

NUNES, D. R. de P.; NUNES SOBRINHO, F. de P. Comunicação alternativa e ampliada para educandos com autismo: considerações metodológicas. Revista Brasileira de Educação Especial. v.16, n. 2, p. 297-312, 2010.

OLIVEIRA, F. I. W. de. Comunicação alternativa. Revista brasileira de educação especial: Marília, 16ed., v.1, p.151-152, Abril-2010.

OLIVEIRA, A. I. A. et al. GAMETERAPIA: POSSIBILIDADES DE AMPLIAÇÃO DO REPERTÓRIO COMUNICATIVO DE UM ADOLESCENTE COM PARALISIA CEREBRAL. In: VI Congresso Brasileiro de Comunicação Alternativa, 6, 2015, Campinas-SP. Campinas. Anais...ISAAC Brasil, 2015, p. 1 - 12.

PAULA, R.; MANZINI, E. J.; DELIBERATO, D. Desenvolvimento de um protocolo para avaliação de habilidades comunicativas de alunos com paralisia cerebral sem oralidade em ambiente escolar. In: IV CONGRESSO BRASILEIRO DE EDUCAÇÃO ESPECIAL, VII ENCONTRO DA ASSOCIAÇÃO BRASILEIRO DE PESQUISADORES EM EDUCAÇÃO ESPECIAL, 3., 2008, São Carlos. São Carlos. Anais...São Carlos: UFSCAR/PPGEEs; ABPEE, 2008, p. 147-147.

QUITERIO, P. L.. Dando voz através da Comunicação Alternativa e Ampliada para promover as Habilidades Sociais na construção da relação com o outro In: IV Congresso Brasileiro Multidisciplinar de Educação Especial. 4., 2007, Londrina, Paraná. Anais: UEL, 2007.

QUITERIO, P. L.; NUNES, L. R. O. L.. O USO DA COMUNICAÇÃO ALTERNATIVA NA AVALIAÇÃO DAS HABILIDADES SOCIAIS DE ALUNOS COM PARALISIA CEREBRAL NÃO FALANTES. In: V Congresso Brasileiro de Comunicação Alternativa, 5, 2013, Gramado. Gramado. Anais...ISAAC Brasil, 2013, p. 1 - 14. 
RI, A. D. et al. Trabalhando a Comunicação Suplementar e/ou Alternativa em um paciente com síndrome de Dandy Walker. In: V CONGRESSO BRASILEIRO DE EDUCAÇÃO ESPECIAL, VIII ENCONTRO DA ASSOCIAÇÃO BRASILEIRO DE PESQUISADORES EM EDUCAÇÃO ESPECIAL, 5., 2012, São Carlos. Anais: UFSCAR/PPGEES; ABPEE, 2012.

REILY, L. Sobre como o sistema Bliss de comunicação foi introduzido no Brasil. In: NUNES, L.R.O.P.; PELOSI, M.B.; GOMES, M.R. (Org.). Um retrato da comunicação alternativa no Brasil: relato de pesquisas e experiências. Rio de Janeiro: 4 Pontos Estúdio Gráfico e Papéis, 2007. v . 2, p. 19-45.

RODRIGUES, V.; CAMPOS, J. A. P. P.; ALMEIDA, M. A. Estudo de caso: efeitos do PECS e Video Modeling na criança com paralisia cerebral. In: VI CONGRESSO BRASILEIRO DE EDUCAÇÃO ESPECIAL, IX ENCONTRO DA ASSOCIAÇÃO BRASILEIRO DE PESQUISADORES EM EDUCAÇÃO ESPECIAL, v.5, 2014, São Carlos. Anais: UFSCAR/PPGEEs; ABPEE, 2014.

SAMESHIMA, F. S. Capacitação de professores no contexto de sistemas de comunicação suplementar e alternativa. 2011. 172 f. Tese (Doutorado em Educação) - Faculdade de Filosofia e Ciências, Universidade Estadual Paulista, Marília, 2011.

SILVA, R. L. M. et al. "PRECISO SER OUVIDO": CARACTERÍSTICAS DA COMUNICAÇÃO DE USUÁRIOS COM PARALISIA CEREBRAL DO NEDETA In: VI Congresso Brasileiro de Comunicação Alternativa, 6, 2015, Campinas-SP. Campinas. Anais...ISAAC Brasil, 2015, p. 1 - 12.

TENOR, A. C.; DELIBERATO, D. ANÁLISE DO USO DE INSTRUMENTO DE AVALIAÇÃO PARA CRIANÇAS USUÁRIAS DE COMUNICAÇÃO ALTERNATIVA. In: VI Congresso Brasileiro Multidisciplinar de Educação Especial. 6., 2011, Londrina, Paraná. Anais: UEL, 2011, p. 1-8.

TOGASHI, C. M. et al. Comunicação Alternativa e a utilização de diferentes modelos de pranchas de baixa tecnologia. In: IV Congresso Brasileiro Multidisciplinar de Educação Especial. 4., 2007, Londrina, Paraná. Anais: UEL, 2007, p. 1-8.

TRUJILLO, F. A. Metodologia da ciência. 3ed. Kennedy: Rio de Janeiro, 1974.

VASCONCELOS, R.. Paralisia Cerebral: a fala na escrita. 1999. 138f. Dissertação (Mestrado em Linguística). Programa de Pós-Graduação em Linguística Aplicada e Estudos da Linguagem. Pontifícia Universidade Católica de São Paulo, 1999.

VEGA, F.; GRÁCIA, M. Comunicação simultânea para melhorar habilidades comunicativas de crianças com deficiência intelectual: trabalho colaborativo com professores. In: VI CONGRESSO BRASILEIRO DE EDUCAÇÃO ESPECIAL, IX ENCONTRO DA ASSOCIAÇÃO BRASILEIRO DE PESQUISADORES EM EDUCAÇÃO ESPECIAL, v.5, 2014, São Carlos. Anais... São Carlos: ABPEE, 2014.

VIGOTSKI. L. S..Pensamento e linguagem. São Paulo: Martins Fontes, 2000. 
WALTER, C.C. de F.. Os efeitos da adaptação do PECS associada ao curriculum funcional natural em pessoas com autismo infantil. 2000. $184 \mathrm{f}$. Dissertação (Mestrado em Educação Especial) - Universidade Federal de São Carlos. São Carlos. 2000.

Avaliação de um Programa de Comunicação Alternativa e Ampliada para Mães de Adolescentes com Autismo. 2006. 107 f. Tese (Doutorado em Educação) Universidade Federal de São Carlos. São Carlos. 2006.

ZIOMEK, M. REHDELFT, R. A. Investigating the acquisition, generalization, and emergence of untrained verbal operants for mands acquired using the Picture Exchange Communication Systems in adults with severe developmental disabilities. The analysis of Verbal Behavior. 24, p. 15-31, 2008.

\section{Correspondência}

Fabiana Lacerda Evaristo - Universidade Federal de São Carlos (UFSCar). Rodovia Washington Luís, km 235, CEP: 13565-905, São Carlos, São Paulo, Brasil.

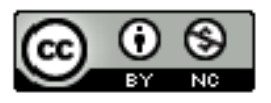

Th is work is licensed under a Creative Commons Attribution-NonCommercial 4.0 International (CC BY-NC 4.0) 